\title{
A MOTOR THEORY OF RHYTHM AND DISCRETE SUCCESSION. I. ${ }^{2}$
}

BV R. H. STETSON, Beloit College.

\section{Method of Experimentation. ${ }^{2}$}

Tables I., II., and V., were prepared from measurements of records taken from up-and-down movements of the hand and arm. The subject held a baton to which a delicate rubber cord was attached. Through guides this cord passed parallel to the axis and close to the surface of the kymograph cylinder. The writing point was attached to the rubber cord itself and a slight torsion was sufficient to keep it pressed against the surface of the drum. The movements were approximately in the same plane, though the records were not materially altered if the baton deviated from the plane. This prescribed path of the baton was the one limitation to the free movement of the subject. The pull of the rubber cord was so slight as to be practically imperceptible when combined with the weight of the baton. By varying the distance of the writing point from the fixed end of the rubber cord, the ratio between the length of the stroke and the length of the record could be varied so that the subject might beat as long a stroke as he pleased. The records taken were all about one fourth to one sixth the amplitude of the actual stroke.

Tables III., IV., VI., VII., were prepared from records taken of the finger tapping on a key, or rubber tambour, and from the movement of the foot in tapping on the floor. The record of the finger was made by a Marey tambour, or an electric marker. In the case of the foot, the heel was kept in position by cleats on the floor, and the foot was made to move up

1 The MS. of this article was received September 3, 1904.-ED.

2 The experimental work on which this paper is based was done in the Beloit Peychological Laboratory recently founded, under the direction of Prof. G. A. Tawney, by the adminiatrators of the Wm. E. Hale Fund. 
and down between guides. The stylus was attached to the sole of the shoe and wrote directly on the kymograph drum. The method of recording the beat of the foot directly was satisfactory ; the friction of the guides could not be detected, and the record was that of a foot tapping time under normal conditions. The usual precautions in dealing with a double kymograph record were taken.

\section{Classification and Definitions.}

\section{Classification of the Various Kinds of Rhythm.}

There are many different forms of the rhythm-experience. One hears of the rhythm of prose, of the rhythm of bird music and of animal calls, of the rhythm of walking and rowing, of the rhythm of simple taps or of 'simple sound series,' of the rhythm of verse, and of the rhythm of music. Wide differences are recognized, and some classification based on the causes of these differences is important for a theory of rhythm.

A common classification is based on the 'content' of the rhythm, a convenient and obvious basis of classification. The simple sound series, or a simple movement series, is assumed as the form of 'pure' rhythm, and all other forms of rhythm are deviations due to the nature of the 'content' which modifies the 'pure ' rhythm.'

The material rhythmized is conceived as an antagonistic force which destroys the regularity and therefore the 'purity' of the rhythm. The more elaborate the 'ideational content,' therefore, the less regular the rhythm and the more must groupings depend on a cause foreign to the rhythm.

By this method rhythms are classified as ( $\mathrm{r}$ ) simple series in which the content is reduced as much as possible and the rhythm is perfectly regular; (2) musical rhythms in which ideational content of a certain type is present; melody and harmony enter in and partly determine the nature of the groupings which the rhythmic forms present, (therefore the rhythm is more irregular, less 'pure' than that of the simple sound series); (3) the

\footnotetext{
'Meumann, E., Phil. Stud., 10, S. 310, etc. Followed by MacDougal, R., PSY. REv., 'o2, 9, p. 476.
} 
rhythm of verse in which a content of a much richer and more independent character has a much greater influence in the formation of the various rhythmic groups, and indeed practically dominates the grouping process and the rhythm per se is at a very low ebb.

The adherents of this system of classification might have carried the process one step further, and found in prose rhythm the complete domination of the ideational content and the consequent complete elimination of the 'pure' rhythm. But it does not then appear how rhythmic prose is to differ from unrhythmic prose.

This classification of rhythms by their ideational content is worthless. It is based on a hasty generalization of the relation of artistic form to artistic content, and when worked out is simply at variance with the facts. Rhythm as a form in music and verse is in the same case with symmetry in the spatial arts, or logical organization in written composition, or the principles of harmony in musical composition. The material does not war with the form and wrench it from its true proportions. In art works involving rhythm, the rhythmic form is not distorted by the material in which it is embodied. It is rather made by that material, and the most elaborate rhythms would be pointless and hard to grasp without 'content.' Anything that happens to rhythm at the hands of the true artist in his treatment of his material will not affect the 'purity' of the rhythm; it will be just as much a rhythm, in every sense, as the barest set of monotonous sounds that was ever clacked out by a laboratory apparatus. As a matter of fact, the artist observes certain requisites of rhythm which the laboratory worker frequently overlooks.

The classification by modifying content does not represent the observed facts. It is a sheer assumption that regularity is the characteristic of the 'pure' rhythm, but granting for the moment that it is, it is easy to show that the three classes, (I) simple sound series; (2) music ; (3) verse, do not show less and less regularity as the theory demands. Owing to the construction of most laboratory apparatus, the simple sound series have usually been objectively regular, but it is easy to prove that very 
wide irregularities can be introduced into such a series without destroying the rhythm. Just as great irregularities are possible as in the case of verse with its rich and definite content. ${ }^{\text {. }}$

According to the classification by ideational content, musical rhythm should be less regular than the rhythm of the simple sound series. But the fact is that it is only in music that a regular quantitative system has been worked out and applied. It is only in music that delicate and complex rhythms demanding minute and accurately differentiated intervals are possible. The many mechanical devices for producing music are witnesses to an exactness in musical rhythm intolerable elsewhere. Instead of being ' modified' and less 'pure' because of an ideational content, musical rhythms are by far the most regular and the most elaborately wrought in the whole range of rhythmic experience.

As to the regularity of the rhythm of verse, it is true that ordinary verse is read with extreme irregularity. And yet this irregularity is in no wise essential to verse rhythm. Series of nonsense syllables and nonsense verses may be, and usually are read with as great regularity as that of the ordinary song, or of the simple sound series. That the ideational content has little to do with the rhythmic impression is apparent when one listens to the reading of verse in a language of which one does not understand a word. In that case the effect is not unrhythmical. We do not miss the content; we do not feel that the factor which determines the unities and the grouping is lost. The verse has not become mere chaos which must be ordered by an unknown content before it can become a satisfactory rhythm. Not at all; one often notes rhythmic peculiarities and beauties not so apparent in a familiar tongue.

Moreover, it is worth noting in the case of these three divisions, that a form may be transferred, without any change, from one to another without losing its satisfactory rhythmic character. If a simple sound series like the striking of a clock is given a set of melodic intervals, the resulting melody does not show any clash between its content and the 'pure' rhythm in which it moves. One method of piano teaching which has

'Stetson, R. H., 'Rhythm and Rhyme,' Harvard Psy. Stud., I, p. 420 ff. 
considerable vogue compels the student to translate his composition into a series of clicks at a mechanical keyboard, and thus to master the technical difficulties before it is played at the piano. The clicks of the mechanical keyboard used are precisely a simple sound series, and the rhythms intended for a musical composition with an ideational content have been translated verbatim into this simple sound series. But the rhythm does not therefore suffer, nor is there any feeling of some lost 'principle of unification.'

The content has something to do with the selection of an appropriate form of rhythm, but, that form once selected, it does not enter as a factor into the rhythm.

In place of the untenable classification based on a modifying content, a classification based on the nature of the rhythms apart from their content is possible. The series of simple sounds, the rhythm of verse, the occupation rhythms like walking, the rhythm of prose, and the rhythm of bird songs are all composed of a single series of sensations.

But it is obvious in the case of many musical rhythms, of some tattooes, of patting time for dancers, etc., that the total rhythmic effect is not produced by a single series of beats.

Certain rhythmic forms, like $\frac{1}{p}$, we cannot produce with

one hand. At least two processes are working side by side in such cases; there is the accompaniment and the melody, or the time-keeping slow beats against the more elaborately figured primary rhythm; often there are three or more distinct lines of beats playing side by side, now coinciding, and now striking alone. This is no mere matter of mechanical convenience in producing the rhythm; it is heard as two or more processes and so noted in our musical scores. It may be objected that many rhythms which are essentially musical have no apparent accompaniment. For example a melody may be given with or without an accompaniment, without essentially changing its rhythm, which is first and last different from the rhythm of verse. $A p$ parently the melody has no accompaniment, but actually the melody has an accompanying rhythm which finds a real em- 
bodiment in the organism of the performer and listener. Every melody has a 'time,' a definite 'takt'; it is in $2-4$ or 3-4 or some other measure type. This definite underlying beat is the simpler, broader rhythm, always observed, and always felt, without which the rhythm of the melody would become a single rhythm. That performer and listener keep this takt means that it must have some physical embodiment, some corresponding movement, for without such movement, no realization of the two beats to the measure or of the three beats to the measure would be possible.

Rhythms, then, may be classified into two large divisions:

r. Rhythms consisting of a single series of beats - e. g., simple sound series, ticking of clock and metronome, verse, prose rhythm, bird songs, occupation rhythms, etc.

2. Combined or concomitant rhythms - e. g., musical rhythms in all their forms, whether accompanied by changes of pitch or not, dancing.

\section{Meaning of 'a Motor Theory of Rhythm.'}

Laboratory investigations recently published ${ }^{1}$ assume the motor explanation. But thus far, little has been done in applying the motor theory to the details of the rhythmic phenomena, and it is by just such a thoroughgoing application that the theory as a principle of explanation must stand or fall. As a general theory, the motor hypothesis needs no defense. Its only competitor was the ' mental activity' theory which is manifestly incapable of explaining the peculiarities of the unit-groups and of the larger groupings. All of the observed facts of rhythm are for it simply arbitrary and unexplained, and its suggestion that content may play a determining part in a rhythm is worse than useless.

When one says that rhythm consists of a series of sensations of movement, or, of a series of sensations of movement in which other sensations (sound, sight, touch) occur precisely as if they were produced by that movement, and that the rhythmic group has the unity of a coördinated action, it is important to know in

\footnotetext{
1 Wallin, J. E., Stud. Yale Pyy. Lab., 'or, 9, p. 130 ; MacDongal, R., Psy. REv., 'N, 9464 and fif.; Miner, J. B., PsY. REv., '03, Mon. Sup., 5 , no. 4, p. 34.
} 
just what sense the word 'consists' is used. It is evident that the mere presence of the movements which might be the basis of rhythmic experience does not imply the perception of that rhythm. The muscular apparatus whereby the sensational basis of rhythm is produced, is developed in the lower animals. Many of their actions are rhythmical, and the parrot probably reproduces rhythmic forms, but no one would credit the animals with a sense of rhythm. The trotting of a horse produces a vigorous rhythm, but one has only to listen to a well-matched team veering slowly into perfect unison, and veering just as gradually out again, to realize that their trotting is rhythmical, but that they have no sense of rhythm. The simplest suggestion would be a 'center' which combines the motor sensations to a rhythmic perception or a rhythmic emotion. But the multiplication of ' centers' never simplified a problem; it is much more nearly in line with what we know of coördination to assume that rhythm is simply a special form of the ordinary coördination of movement-experience. The Wundtians, who insist that rhythm is primarily an affective experience, can always treat the movement basis of rhythm as Stumpf ${ }^{2}$ has treated the Lange-James theory of the emotions, insisting that there is an antecedent central process involved.

The question whether the affective aspect of the rhythm is the essential aspect is really part of a much larger question. If all forms of artistic synthesis, and indeed of any unity, are affective in character, then rhythm is to be so classed. If symmetry, and the metaphysical demand that the world be one, are primarily felt and not perceived, then rhythm is felt and not perceived. The same concrete rhythm may have at different times all shades of affective coloring from pleasant to disagreeable, and must pass the indifference point. Any series of acts is capable of just such changes in affective coloring. To the writer, the simplest form of the rhythm-experience seems simply a perception of a peculiar type of likeness and repetition in a movement-series. Whether or not it is primary no one can deny the importance of the emotional in rhythmic experience.

'Stumpf, C., Zeitschr.f. Psy. 4. Phys. d. S. org., '99, 21, 8. 64-5. 
It remains to consider in view of the motor interpretation the distinction between rhythms when merely perceived and when produced by the subject.

The movements involved in the production of a rhythm are always at hand as a basis for the experience of the produced rhythm. But one has as vivid and satisfactory a sense of rhythm when one merely hears (or sees, or feels) the series. Where are the movements at the basis of such a 'sensory' rhythmic experience? The body is provided with muscles capable of producing rapid and varied movements not visible to ordinary observation; among these one looks most naturally to those organs which have to do with the production of rhythm. Many musicians keep the takt by tapping the foot, and strains of the muscles of the leg often constitute the silent rhythmic response. Others tend to move the head or the trunk in time; careful observation of a concert audience will show both these types of motor rhythmization. But the most important natural rhythm-producing apparatus is the vocal apparatus. Musicians are frequently trained to count, and suppressed counting is frequent. The tongue is extremely mobile, and the muscles of respiration play a frequent part in rhythmization. The writer finds rapid series rhythmized by slight movements of the muscles of the tongue and perhaps of the throat, in conjunction with the expiratory muscles which mark the main accents. Every rhythm is dynamic; it consists of actual movements. It is not necessary that joints be involved, but changes in muscular conditions which stand in consciousness as movements are essential to any rhythm, whether 'perceived' or 'produced.'

In developing a motor theory of rhythm there are certain principles of explanation that are barred. They have frequently found a place in discussions assuming the motor basis, but they will not play any part in the present attempt at a motor explanation.

Analogies drawn from space do not help. A pause is frequently represented as a dividing space; and often the pause is supposed to separate, and therefore to unify the groups.' But unless motor changes can be shown to take place during the pause, the pause has no significance.

${ }^{1}$ Wallin, loc. cit., p. 92 ; Miyaki, I., Stud. Yale Psy. Lab., '02, ro, pp. 16 ff. 
Nor does the logical supposition that as single beats are combined into unit-groups, so unit-groups may be combined into phrases, phrases into periods, etc., constitute a legitimate method of explaining the larger groupings of rhythms (Wundt and Meumann). As will be seen later, the nature of the larger groups is entirely different from the nature of the unit-groups.

Judgments of temporal equality or inequality play no part in the rhythm experience. ${ }^{1}$ The time judgment is much too vague to determine rhythmic intervals, and accurate judgments of time founded on rhythms are secondary and derived.

Explanations based on physiological rhythms, such as that of the heart, ${ }^{2}$ or of a supposed rhythm of the nervous discharge, ${ }^{3}$ can avail nothing. Like the 'mental activity,' such explanations fail to account for the wide variations in tempo, and for all the peculiar facts of the unit-group and of the larger groups.

Imagery, or ' central motor discharges,' " or 'mental beats's have often been invoked as possible vehicles for a rhythm. Imagery undoubtedly does play an auxiliary part in any rhythm, but that it can actually constitute a rhythm seems more than doubtful. A theory of rhythm in which the essential process is phrased in strictly peripheral terms is safe from the vagueness and uncertainty of the concept of mental imagery. Such a theory could be easily translated into terms of 'central motor discharges'-if their existence should be demonstrated, and rhythms of that type detected.

\section{Single Rhythms.}

1. Nature of the Movement-Cycle of Any Rhythm.

A movement may be perfectly regular, uniform, and recurrent and yet not give the impression of rhythm. If one moves the hand or arm in a circle, the hand may be made to pass a point in a circle much oftener per second that the tempo of the slower rhythms requires, and yet there will be no feeling of rhythm

${ }^{1}$ Squire, Mrw. C. R. (following Ettlinger), Am. J. Psy., 'D, 12, p. 541.

'Hallock, Miss M., Pop. Sci. Monthly, '03, 63, Sept., p. 425.

- MacDongal, R., loc. cit.

- Miner, loc. cit., p. 33.

6 Wallin, loc. cie., p. 130. 
so long as the hand moves uniformly and in a circle. In order to become rhythmic in the psychological sense, the following change in the movement is necessary: The path of the hand must be elongated to an ellipse; the velocity of the movement in a part of the orbit must be much faster than in the rest of the orbit; just as the hand comes to the end of the arc through which it passes with increased velocity, there is a feeling of tension, of muscular strain; at this point the movement is retarded, almost stopped; then the hand goes on more slowly until it reaches the arc of increased velocity. The rapid movement through the arc of velocity and the sudden feeling of strain and retarding at the end of this rapid movement constitute the beat. In consciousness they represent one event, and a series of such events connected in such a movement-cycle may be said provisionally to constitute a rhythm. Every rhythmic beat is a blow. The origin of rhythm, as Bücher has suggested, was in forms of concerted work which required blow on blow. That is possibly the genetic reason why the beat, the blow, is the primary thing in the rhythm-consciousness. In all forms of activity where a rhythm is required, the stroke, the blow, the impact, is the thing ; all the rest is but connection and preparation. The movement in striking a quick blow or in beating a rhythm may be represented by $A$ and $B$ in the movement curve of Fig. 5 in which $A$ represents the lifting of the member and $B$ the rapid blow.

There is, then, a radical difference between the two phases of the rhythm-movement. The first great difference lies in the velocity of the two parts of the movement. In $B$ of Table I., are given the velocities of the two phases of the movement in the case of three subjects beating rhythms as rapidly as possible. The velocity of the beat stroke is always two or three times greater than the velocity of the back-stroke, and the total relaxion-phase is always at least three times as long as the contraction-phase. A second difference between the two phases lies in the control which the subject has over the moving member; once the beat stroke is started, like a released spring the limb flies to the end of the stroke, and is in no wise subject to regulation; during the back-stroke on the over hand, the movement 


\section{TABLE I.}

VELOCITY OF THE DOWN-STROKE AND OF TEH UP-STROKE.

$A$. Velocity of the Down-stroke in very slow movements. Movement of the foot as slow as possible.

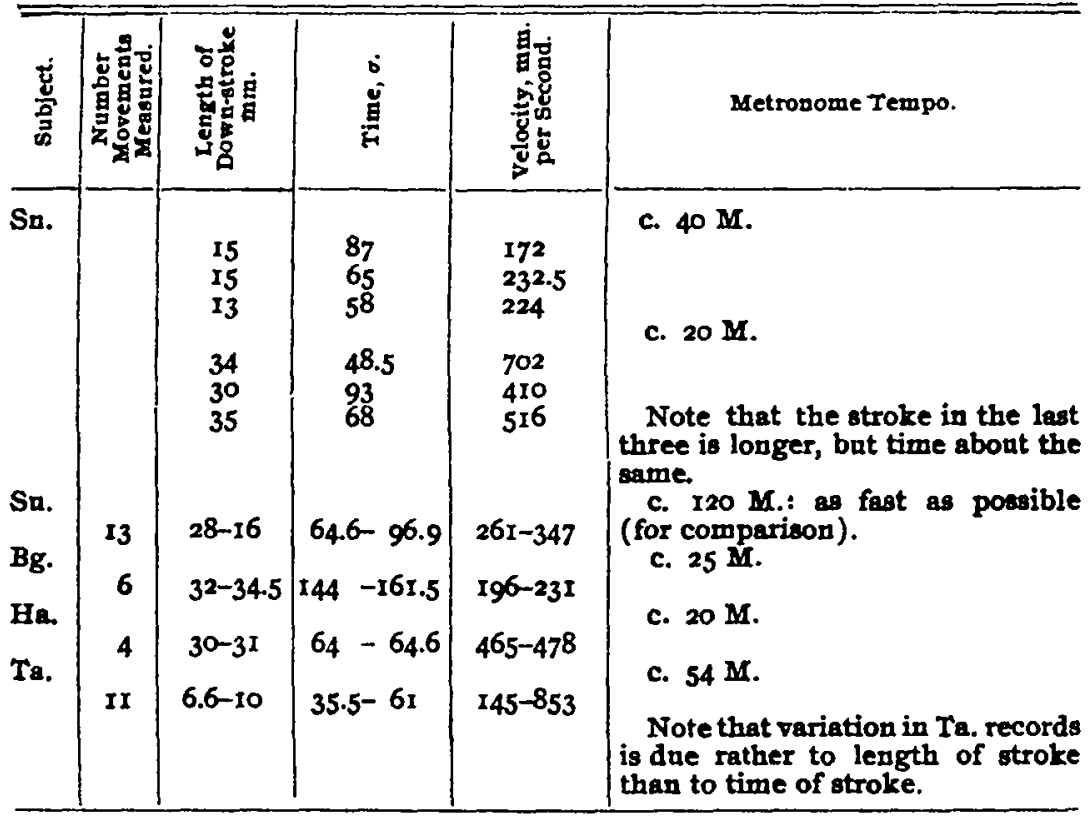

$B$. Velocity of the down-stroke and up-stroke. Free movement of the hand and arm, as fast as possible.

\begin{tabular}{|c|c|c|c|c|c|c|c|c|}
\hline 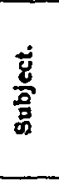 & 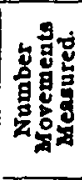 & 递 & 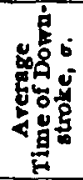 & 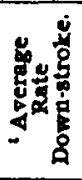 & 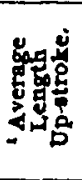 & 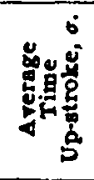 & 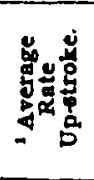 & $\begin{array}{l}\text { Metronome } \\
\text { Tempo. }\end{array}$ \\
\hline \multirow[t]{3}{*}{ Th. } & $\begin{array}{l}I \\
I \\
I \\
I \\
I \\
I\end{array}$ & $\begin{array}{l}81 \\
63 \\
72 \\
70 \\
72 \\
48\end{array}$ & $\begin{array}{c}113 \\
80.7 \\
97 \\
103 \\
96.8 \\
96.8\end{array}$ & $\begin{array}{l}742 \\
807 \\
774 \\
720 \\
774 \\
516\end{array}$ & $\begin{array}{l}\mathbf{8 1} \\
55 \\
74 \\
77 \\
54 \\
53\end{array}$ & $\begin{array}{l}158 \\
139 \\
158 \\
177.5 \\
145 \\
171\end{array}$ & $\begin{array}{l}516 \\
419.6 \\
484 \\
452 \\
387.5 \\
323\end{array}$ & c. $210 \mathrm{M}$. \\
\hline & 6 & 67.6 & 96.8 & 730 & 66 & 161.5 & 436 & \\
\hline & 9 & 75 & 78.8 & 1033 & 74.3 & 184 & 426 & c. $210 \mathrm{M}$. \\
\hline St. & $\begin{array}{l}4 \\
4\end{array}$ & $\begin{array}{l}46 \\
51\end{array}$ & $\begin{array}{l}84 \\
80.7\end{array}$ & $\begin{array}{l}595 \\
646\end{array}$ & $\begin{array}{l}46 \\
49\end{array}$ & $\begin{array}{l}129 \\
126\end{array}$ & $\begin{array}{l}390 \\
390\end{array}$ & c. $240 \mathrm{M}$. \\
\hline Bi. & 14 & 42.6 & 48.8 & 952 & 43 & I38.7 & 336 & c. $260 \mathrm{M}$. \\
\hline
\end{tabular}

1 The lengths tabulated and therefore the rates are functions of the actual lengths. The actual stroke was about five times the recorded values. 
In these very rapid movements the time of the up-stroke is much longer than the time of the down-atroke, and between the end of the up-stroke and the beginning of the down-stroke there is always a curve not here recorded, equal in duration to the down-stroke, so that the total duration of the 'relaxation phase is at least three times the duration of the 'contraction phase.'

may be regulated or changed at will. A third difference is noticed when two rhythms are beaten at the same time; the subordinate pulses of the one rhythm never occur during the beatstroke of the other rhythm.

Nature of the Movement Beat-stroke. - However fast or slow the rhythmic movement, this high velocity is characteristic of the beat-stroke. In Table I., $A$, are given the duration values and the velocities of the beat-strokes in the slowest possible rhythms. It will be noted in the case of all four subjects that the duration of the beat-stroke is never more than too sig., and that the velocity is dependent on the length of the beat-stroke and not on the tempo of the rhythm. Under special limiting conditions the duration of the beat-stroke may be as long as 400 sig., ${ }^{1}$ but in all the records of freely-executed rhythms there are no beat strokes longer than 125 sig. and the velocity of the beat-stroke for the range of lengths which rhythmic movements present seems to be independent of the length of stroke. It is probable that if the movements were to be greatly exaggerated, differences of duration would appear; rapid movements of the eye show that the duration of the movement is affected by its length, though it does not vary directly. The duration of the beat-stroke is strikingly uniform, and is independent of either the tempo of the rhythm or the length of stroke. The clearest discussion of the events in such a rapid stroke brought to a sudden stop by the musculature, or by an obstacle, or by both, is found in Richer's discussion of human locomotion. ${ }^{2}$ Richer divides all movements into three classes:

r. The two muscles or sets of muscles $(i . e .$, the antagonists, as the flexors and extensors, which produce the movement) contract simultaneously. (Ordinary type of slow movement.)

'Cf. Table II.

'Traite de Physique Biologique, D'Arsonval et Autres, Paris, 'or. I., ' Locomotion Humaine,' p. 156 et suite. Richer, P., 'Note sur la contraction du muscle quadriceps dans l'acte de donner un coup de pied,' Soc. de Biol., '95, Mars. 23. Athanasius, M. I., gives graphic records of similar movements ob-

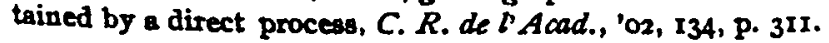


2. The one contracts, the other is passive. (Very excep tional type.)

3. The one contracts while the other relaxes and lengthens.

The rapid stroke in question is a form of this third type. This particular form of light, rapid, and sometimes repeated blow, Richer calls ' ballistic.' By the study of instantaneous photographs he has determined that at the beginning of the stroke the positive muscle-set (extensors in case of the fore-arm) are suddenly contracted, but that by the end of the first third or first half of the movement, the contraction of the positive muscleset has ceased, the positive muscles relax, and the limb is carried past this point by inertia alone. If the blow is delivered in the air, the negative muscle-set (in case of fore-arm, the flexors) contracts and stops the stroke. In rapidly repeated strokes of this kind, the member is returned to the original position by a similar sudden contraction of the negative muscleset, which relaxes in the middle of the blow, and the stroke is in turn checked by the positive muscle-set. Thus the limb is thrown back and forth, and caught in turn at the limits of its movement by the positive muscle-sets. Such a battledore-andshuttlecock type of movement is very aptly termed 'ballistic.' Rieger has recently described the same type of movement,' under the name of 'movements with elastic rebound,' but his form of record does not permit him to observe the action of antagonistic muscles as clearly as has Richer, and his concept of ' elastic rebound' is a better description than explanation of the movement. Rieger is aware however, that the beat-8troke of such a movement and its back-stroke make but a single unity in consciouness, and he promises to apply the principle of such a movement to the concept of accent in a future discussion." As for the mechanism of innervation in such movement, Richer has no suggestion. Sherrington suggested ${ }^{3}$ that ' reciprocal innervation' was the result of a fundamental structure of the muscle apparatus in antagonistic muscle-sets, but as Du Bois Reymond" points out there is no such fundamental grouping of

i Rieger, C., 'über Muakelenatande,' Zschr. f. Psy. w. Phys. d. S. org., 'o3, 32, S. 384 ff.

- Loc. cit., S. $38 \mathrm{q}$

- Proceedings Royal. Soc., '97, p. 415.

- Da Bois Regwond, R., Specielle Muskelphysiologie, Berlin, '03, S. 243. 
muscles into permanent antagonistic sets as the theory would require. Each set may be now antagonistic now synergic, and the rôle of muscles in the varied movements in which they take part is far too complex to admit of any fundamental neurological law of ' reciprocal innervation.' That such a reciprocal innervation does take place in the ballistic movement is evident, but it must be a type of coördination dependent on higher centers, and capable of all sorts of rearrangement, like other forms of comples muscular action and movement. What furnishes the precise signal for the action of the negative muscles in catching the limb tossed to them by the positive set? The case is different from that of an ordinary slow movement, where both sets of muscles are contracted, and the various inflowing kinæsthetic sensations check and guide the contractions of the muscles involved. The movement is very rapid; during the stroke the sensations are extremely vague, and as the experiments discussed later will show, frequently mislocated both in time and space. It does not seem possible that any event after the sudden contraction and relaxation of the positive muscle-set can be the cue for the contraction of the negative muscle-set. It is probable that the cue which sets the negative set to contracting is the contraction of the positive muscleset itself. The brief interval between the positive contraction and the negative contraction, from 30 to 120 sig., is no more than time for a nervous impluse to be generated and reach the negative muscle-set. ${ }^{1}$ The fact that time of the beat-stroke does not vary with the length of the beat-stroke, nor with the tempo of the rhythm favors this automatic connection of positive and negative muscles in the ballistic movement. It is experience alone which teaches us to guide the ballistic stroke. This experience is summed up in images of the movement (visual and 'motor'), which gauge the intensity of the positive muscle-set, and the appropriate contraction of the negative muscle-set follows inevitably.

An obstacle against which the limb strikes does not affect the character of the movement; at the end of the normal inter$\mathrm{val}$ the negative muscle-set contracts and withdraws the limb, as

' CF. Hof bener, I., Arch.f. d. ges. Physiol. (Pfüger's), '97, 68, S. 553. 
if the limb had shot to the end of its course unimpeded. It is simply as if the lower part of the oscillation had been cut off by the obstacle, and its place taken by a pause at the obstacle. If one closes the eyes and beats a rapid rhythm with the arm and hand, at first in the air, and then approaches an obstacle whose position is not exactly known until the hand strikes the obstacle

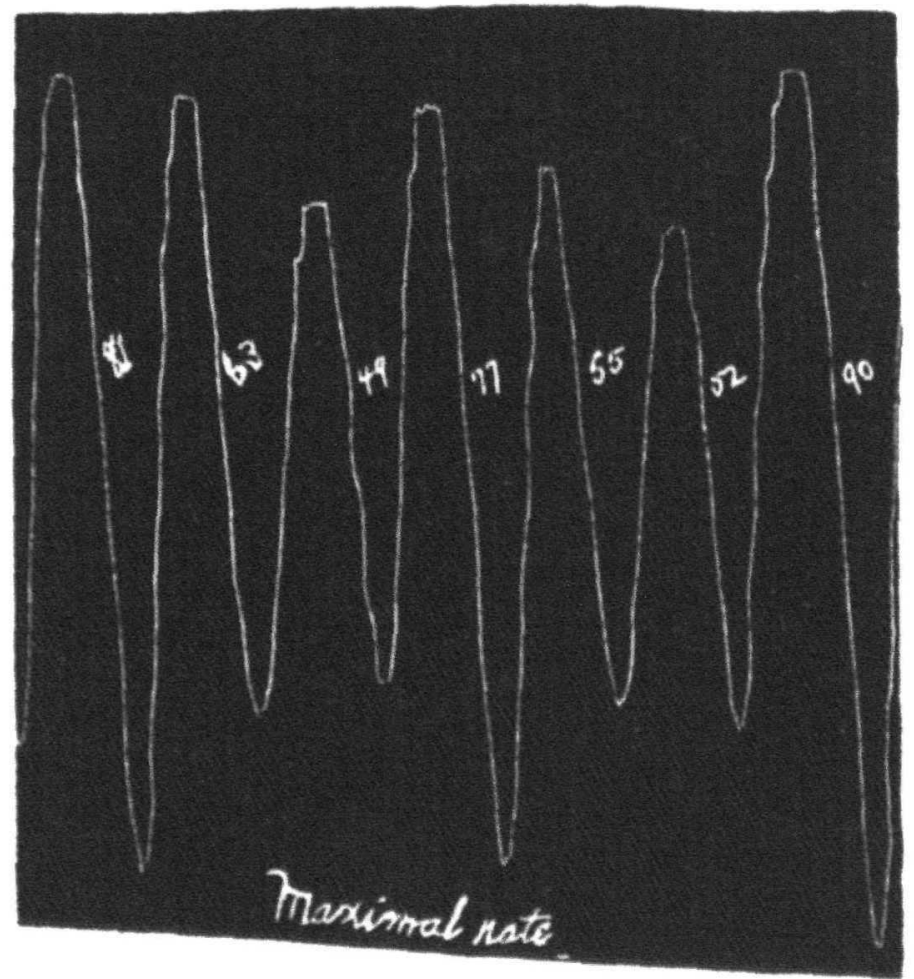

F IC. I

at each beat, one will find that the character of the movement and of the rhythm is quite unchanged by the intervention of the obstacle.

Nature of the Movement in the Back-stroke. - The relaxation-phase is not as invariable as the contraction-phase. In rapid beating the back-stroke begins immediately on the close of the beat-stroke; the record of such a rhythm beaten in the air shows a sharp angle between the beat- and back-stroke. 
But, however rapid the rhythm, the velocity of the back-stroke is always much less ( $c f$. Table I.), and at the end of the backstroke, at the upper limit of the movement, there always appears a rounded curve which is very different from the sharp point at the lower limit.

In such rapid movement it is possible that the limb is driven back by a contraction of the negative muscle-set without any action of the positive-set. If so, the positive muscle-set must come gradually into action during the upper part of the backstroke, for the rounded part of the curve at the upper limit of the most rapid movements can only mean that both sets are contracted, moving the limb slowly or holding it at rest just before the beat-stroke. During a slow rhythmic movement the limb often remains at the lower limit for some time, as if the negative contraction just balanced the momentum of the limb or the residual contraction of the positive muscle-set. In movements with great force at slow rates, it is certain that the positivemuscles are brought into play at the bottom of the beat-stroke, and coöperate with the negative muscle-set during the earlier part of the relaxation period in holding the limb. After this condition of rest at or near the lower limit of the movement for a longer or shorter interval, a very slow rise takes place (this may be modified in combined rhythms, as may be noted later) and the slow round of the curve at the upper limit shows that the two sets of muscles balance each other for a perceptible time just below the beat-stroke.

Although the phenomena of the relaxation-phase are thus somewhat variable, the relaxation-phase is a perfectly definite and essential process. The form of the rhythmic movement may be changed so that the limb does not return during the relaxation-phase to the upper limit for the next beat-stroke, but instead, the next beat-stroke starts from the point where the last beat-stroke ended. Measurements of records of such movements are given in Table II. The subject was directed to beat up and down with a baton, but to make a beat both on the down-stroke and on the up-stroke. The result is that every other beat-stroke occurs in the opposite direction from the intersening beat-stroke, and there is no back-stroke. What of the 
process which the back-stroke represents in a rhythm-movement? On examining the records one sees that there is a long interval between the beat-strokes and during this interval the limb is at rest. (The pause is represented by a straight line on the moving kymograph cylinder.) (Cf. Fig. 2.)

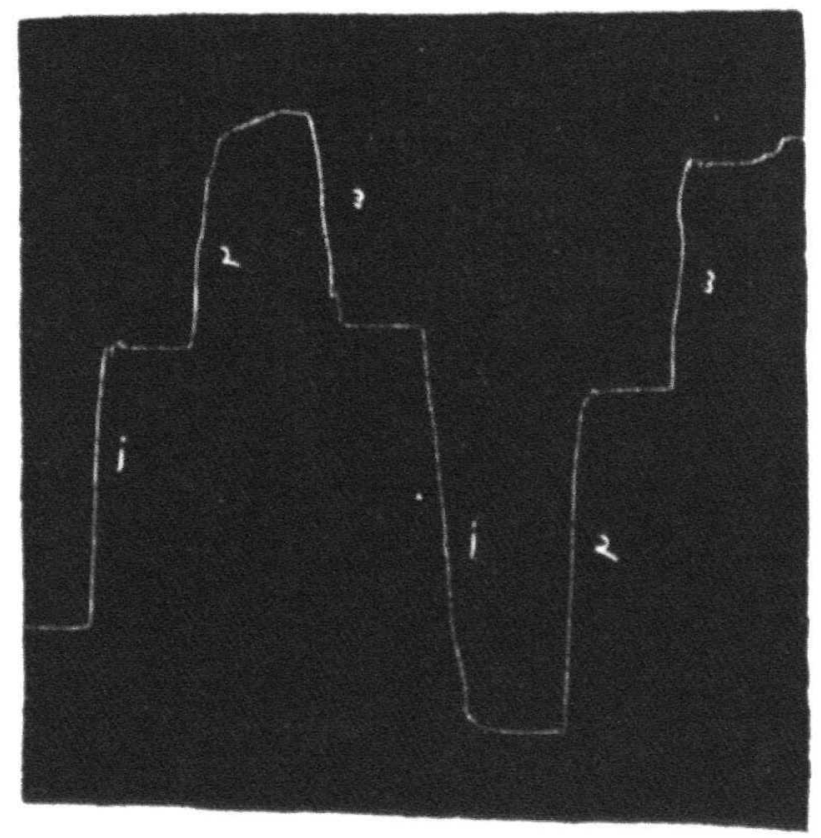

F1G. 2.

The duration of this pause appears from the measurements much longer than the beat-stroke, averaging from two to three times the duration of the beat-stroke. (Cf. Table II.)

The records of the three subjects were taken at medium and fairly rapid tempos, but the tempo does not affect the relative length of the pauses. After the experiments were performed on which Table II. is based, records of Rieger's were found which show the same relations at the fastest possible tempos. The records were taken for a different purpose, but the conditions are the same, and the movement was rhythmical. ${ }^{1}$ At this maximal tempo, Rieger's figures show that at least Loc, cit., S. 388 . 


\section{TABLE II.}

Comparison of the durations of the 'relaration'- and 'contraction'phases of a series of movements in which there is a beat both on down-stroke and np-stroke; these both become 'contraction'-phases and the parwes between them are the relacation-phases.

\begin{tabular}{|c|c|c|c|c|c|c|}
\hline 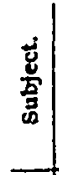 & 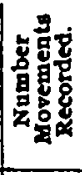 & 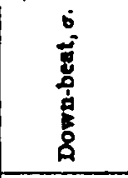 & $\begin{array}{l}\dot{0} \\
\dot{0} \\
\dot{y}\end{array}$ & $\begin{array}{l}\dot{b} \\
\frac{\overrightarrow{8}}{8} \\
\frac{d}{b}\end{array}$ & $\begin{array}{l}\dot{8} \\
\dot{8}\end{array}$ & Metronome Tempo. \\
\hline Th. & $\begin{array}{l}\text { I } \\
I \\
I \\
I \\
I \\
I \\
I \\
I \\
I \\
I\end{array}$ & $\begin{array}{l}355 \\
355 \\
355 \\
323 \\
291 \\
387.5 \\
355 \\
226 \\
387 \\
355\end{array}$ & $\begin{array}{l}387.5 \\
387.5 \\
484 \\
484 \\
452 \\
613 \\
419.5 \\
419.5 \\
452 \\
355\end{array}$ & $\begin{array}{l}193.5 \\
226 \\
193.5 \\
226 \\
226 \\
161.5 \\
226 \\
291 \\
226 \\
226\end{array}$ & $\begin{array}{l}452 \\
355 \\
387.5 \\
484 \\
387.5 \\
419.5 \\
484 \\
323 \\
452 \\
484\end{array}$ & $70 \mathrm{M}$. \\
\hline St. & 12 & Av. 317.5 & Av. 312 & Av. 196.5 & A. 201.6 & $\begin{array}{l}82 \mathrm{M} \text {. Very little variation } \\
\text { in values. }\end{array}$ \\
\hline $\begin{array}{l}\text { Bi. } \\
\text { Th. }\end{array}$ & $\begin{array}{r}12 \\
8\end{array}$ & $\begin{array}{l}297 \\
29 t\end{array}$ & $\begin{array}{l}490 \\
492.5\end{array}$ & $\begin{array}{l}193.6 \\
121\end{array}$ & $\begin{array}{l}323 \\
489\end{array}$ & $\begin{array}{l}76 \mathrm{M} \text {. Very little variation } \\
\text { in values. } \\
78 \mathrm{M} \text {. Verg little variation } \\
\text { in values. }\end{array}$ \\
\hline
\end{tabular}

Comparison of the durations of the 'relaxation'- and 'contraction'. phases of a series of movements in which there is a down-beat, then a second down-beat, then an up-beat, then a second up-beat, etc. ( $c f$. cut 2 ).

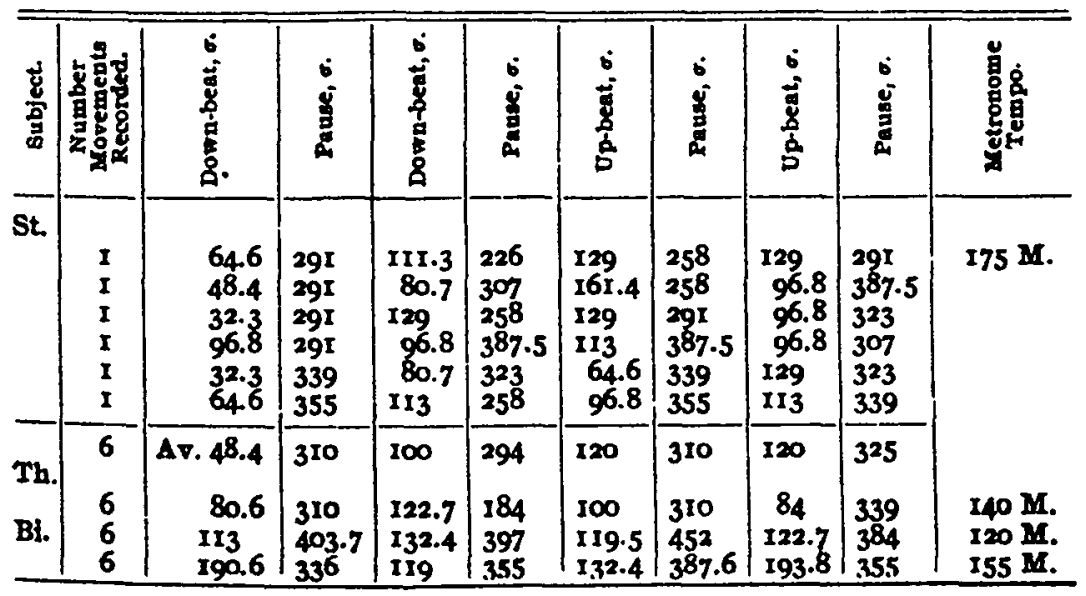


three fourths of the time was consumed in the relaxation-phase of the rhythm-movement. It is apparent that some preparatory process is necessary after one beat-stroke, before another beatstroke can be made. Rieger's figures go to prove that this preparatory process takes more time when performed with the limb at a standstill, than when the limb is in motion as in the usual back-stroke.

This preparatory process must consist of a change in muscular tensions, since the limb is practically at rest. At the end of the beat-stroke, the negative muscle-set contracts sharply; in rapid beating at least, there is also a contraction at this point, or a residual tension, in the positive muscle-set. Except possibly in very slow beating, the contraction of the negative muscle-set cannot be so proportioned as to just balance the momentum of the limb, and tension in the positive muscle-set is necessary if the limb is to come to a standstill at the close of the beat-stroke. During the long pause which takes the place of the ordinary back-stroke the contraction of the negative muscle-set gives way to relaxation and the tension in the positive muscle-set becomes very slight. This is the poised condition just before the second beat-stroke.

The relaxation-phase alone is subject to control. Changes in tempo are due to voluntary hastening or retarding during the relaxation process. This fact is of considerable importance in conducting. A chorus or orchestra depend quite as much on the back-stroke as they do on the beat-stroke for direction. Conducting at the organ or piano is always unsatisfactory, and an angular style of beating which suppresses the back-stroke is almost as ineffective.

The events, then, in any rhythmic movement-cycle producing the simplest possible series of beats is as follows: At the beginning of the beat-stroke there is a sharp contraction of the positive muscle-set, setting the limb into rapid motion, during the earlier part or the first half of which the contraction of the positive muscle-set gives way to relaxation; this contraction-relaxation process in the positive muscle-set is the cue for the automatic contraction of the negative muscle-set; at the end of the flight of the limb, the negative muscle-set contracts sud- 
denly and automatically and arrests the limb; this contraction of the negative muscle-set works against the momentum of the limb and a possible residual or active tension of the positive muscle-set; this ends the contraction-phase. After the contraction of the negative muscle-set has overcome the resistance at the bottom of the stroke, the contraction grows rapidly less, any tension between the two muscle-sets is reduced, and the condition of a very slight tension between the two muscle-sets, with the limb poised, is reinstated; this is the condition at the beginning of the beat-stroke (in the normal movement-cycle, the limb is raised at a varying velocity to its original position during the readjustnent of the relaxation-phase) and the close of the relaxation-phase.

This movement-cycle is usually given its form and limits by a rather definite image of the movement. The upper limit with many subjects does not seem to be reached by a uniform movement through a certain time or during a certain definite change of tensions, for these subjects raise the limb at varying points in the cycle to the proper height and leave it at rest, awaiting the beat-stroke. But in itself this image of the movement would not give rise to any feeling of movement, or any impression of rhythm.

In the case of a rhythm beaten against a heavy resistance the figure previously published ${ }^{1}$ is probably an approximate representation of the movement, but for the normal, freely beaten rhythm, the writer now considers the accompanying Fig. 5 a more accurate representation of the process ( $f f$. Fig. 5).

Temporal Relations which Depend on Mere Accent. Aside from the temporal relations of the typical unit-groups to be considered later, there are certain temporal relations which are to be referred directly to the nature of the movement-cycle of the single beat. The well-known lengthening of the accented beat, and the 'pause' which precedes the accented beat are due to modifications of the cycle. There are two ways in which the increased force of an accented beat may be obtained; either the length of the stroke may be increased (just as the upright-piano action is arranged to give degrees of loud and soft

'Rhythm and Rhyme,' Harvard Psy. Stud., I, p. 454. 
by varying the length of the hammer-stroke), or the path of the limb may be unchanged and the contraction of the positive muscle-set more powerful. As greater length is usually associated with intenser contraction, probably both occur in untrammeled beating. Either of these ways will slightly modify the temporal relations of the resulting beats.

Although the time of the beat-stroke is little changed if the stroke is lengthened, the limb must be raised higher than usual, and this must add to the interval preceding. If there were any increase of the duration of the beat-stroke, that would be added to the preceding interval, for the ordinary methods of measurement make the end of the beat-stroke, or the limiting sensation the point of departure.

If the limb, in beating rhythmically, does not impinge on an obstacle, the accented beat will descend lower than the average beat, because its greater momentum will carry it farther. It will take more time for the relaxation process following the heavy beat, for higher strains must be reduced to the poised condition, and in untrammeled beating the limb must be raised through a longer back-stroke to the usual upper limit of the movement. As noted above (Fig. I), an obstacle against which the limb strikes does not change the character of the movement; the action of the muscles is the same as if the limb had shot down to the end of the interrupted stroke. But it is customary to measure the beat from the point where the limb first touches the obstacle, 80 that a rhythm in which the accent is determined largely by increased contraction with the limb striking an obstacle will show by the ordinary method of measurement a decided increase in the length of the interval following the accented beat. The accented movement must always take more time however the increase is distributed before and after the beat by the particular method of measurement chosen. 